\title{
MULTITURN SIMULATION OF COHERENT BETATRON RESONANCE WITH SPACE CHARGE
}

\author{
M. D'yachkov, R. Baartman and F.W. Jones \\ TRIUMF, 4004 Wesbrook Mall, Vancouver, Canada V6T 2A3
}

\begin{abstract}
The space-charge analysis code TRANSOPTR, paired with the tracking and simulation code ACCSIM, have been applied to study the half-integer betatron resonances in a high-intensity proton ring. We investigate the relationship of coherent-mode frequencies with the incoherent (Laslett) tune as a function of beam intensity, and the existence of space-charge intensity limits due to detuning of the coherent modes onto a resonance. Details of the computational model and solution methods are also presented.
\end{abstract}

\section{INTRODUCTION}

In this study we apply macroparticle simulation techniques to investigate a case where space charge destabilizes a circulating beam by moving it onto a betatron resonance. We use a realistic synchrotron lattice (FNAL Booster) and explore the implications for intensity limitations in operating the ring. In such simulations it is often difficult to isolate a particular physical phenomenon: the results are frequently complicated by mismatch oscillations and beam redistribution, which have relatively long relaxation times compared with the computing times available.

Therefore, we have paid particular attention to initial conditions. We solve the envelope equations to establish a stable set of initial conditions in a given intensity range. This helps both to validate the space-charge simulation and to provide a clear benchmark against which to measure resonant growth. To this end we set up a software framework to identify cases, calculate lattice and beam-distribution parameters, perform the multiparticle simulations, and analyze the results. The framework employs some Perl scripts, the analysis/optimization code TRANSOPTR, and an enhanced version of ACCSIM. We describe the software tools and results in the following sections.

\section{TRANSOPTR}

TRANSOPTR[2] differs from TRANSPORT and other codes in that the beam transport is coded directly in a computer language (FORTRAN in this case). This allows for much greater flexibility in fitting, both in terms of parameter definition and fitting criterion. As originally written, it was intended for beam transport lines. However, by modifying it to allow fitting of the input beam to achieve a matching condition, it is now also capable of treating synchrotrons and storage rings. Space charge is handled not by inserting pseudo-lenses meant to represent continuous space charge defocusing, but rather, the equation of motion of the beam envelope is solved directly with a Runge-Kutta routine, using the so-called infinitesimal transfer matrix. This matrix $F(s)$ is defined as $(T-I) / d s$ where $T$ is the transfer matrix from $s$ to $s+d s$ and $I$ is the identity matrix. With this definition one has for individual particles $d \mathbf{x} / d s=F \mathbf{x}$ Here $\mathrm{x}$ denotes the 6-dimensional phase space vector. Beams of particles are conveniently represented by the so-called sigma matrix; the elements of which represent second order moments of the beam: $\sigma_{i j}=\left\langle x_{i} x_{j}\right\rangle$. The sigma matrix transforms through the system according to the equation[1]

$$
\frac{d \sigma}{d s}=F \sigma-\sigma F^{T}
$$

where $F^{T}$ is the transpose of $F$. Linear space charge effects are easily included by adding the linear parts of the space charge forces to the relevant elements in the $F$-matrix of the element being traversed. For systems with no energy spread and no $x-y$ coupling elements, it can be shown[1] that eqn. 1 reduces to the well-known Kapchinsky-Vladimirsky equations.

Optimization is handled by a simulated annealing routine from Numerical Recipes which is built on top of a 'simplex' algorithm. This is especially useful when fitting 3 or more parameters. For few parameters, the initial 'temperature' can be set to zero, in which case the optimization is by the 'simplex' method. See ref. [3]. In general for a synchrotron, one needs to fit $\alpha$ and $\beta$ in both $x$ and $y$, as well as the dispersion function and its derivative. In a straight-forward manner, such a 6-parameter fit yields space-charge-modified values of all beam parameters, including momentum compaction[4].

\section{ACCSIM}

ACCSIM[7] is one of a small number of codes developed to support multiturn space-charge tracking in synchrotrons. Within the conventional "field-solve/particlepush" integration mechanism it employs an efficient $2 \frac{1}{2} \mathrm{D}$ model, allowing rapid solution of the space-charge field, and matrix/thin-lens transport. As with a similar code Orbit[8], the basic magnet lattice is described in the standard way using the MAD format and is preprocessed by a lattice code DIMAD (Orbit uses MAD) to find the optical functions, transfer matrices, and other parameters. The output file from DIMAD is then parsed by ACCSIM to extract this information for use in the actual tracking/simulation run.

Offloading much of the preparatory work to a preexisting lattice code has brought many benefits including 
the rapid development and commissioning of the optics and transport functions within ACCSIM. For space-charge tracking, however, there is a negative impact in that relatively short, regular transport steps are necessary in order to perform the frequent field-solves needed for accurate results. This entails the subdivision of drifts and magnets into slices and making a new lattice definition from these shorter drift and magnetic elements, so that ACCSIM will have access to the transfer matrix for each slice. The subdivision process is tedious and error-prone and, as well as being a deterrent to potential ACCSIM space-charge users, it makes it impractical to quickly change lattice parameters and/or the tracking step size.

For these reasons it was inevitable that ACCSIM become a stand-alone program that is able to (1) read and parse a MAD-format lattice definition and associated parameters, (2) compute all transfer matrix coefficients for lattice elements, (3) solve for the matched optics and tunes of the ring, (4) according to a tracking step size, subdivide lattice elements into slices and compute transfer matrices for each slice. The necessary additions to the code are now mostly in place and after some further refinements will be included in the next release, ACCSIM 4.0. For the present study, using this expanded version allowed a much simplified process of generating ACCSIM runs from TRANSOPTR output.

\section{HALF-INTEGER RESONANCES}

The evolution of the second moments of the beam is calculated by TRANSOPTR under the assumption of constant rms emittances and linear space charge forces. So the calculated matches actually only apply to KV distributions. However, in synchrotrons and storage rings, the space charge tune shift is small compared with the tune, and emittance growth per turn is small even when betatron resonances are important. Under these conditions, matched beams calculated by TRANSOPTR are relevant and indeed simulations with ACCSIM bear this out.

We have chosen to work with the FNAL booster lattice. The intention is to investigate the half-integer stopband at $\nu_{x}=13 / 2$, when space charge depresses the envelope eigentune to 13 . A plot of matched $\beta$-functions vs. circulating current is shown in Fig. 1, for tunes $(6.7,6.8)$ and a single thin lens to drive the $13 / 2$ resonance. We note that the resonance occurs not when the incoherent tune is near 13/2, but when the envelope tune is. The two tune shifts differ by a factor of 0.68 , in agreement with theory[6, eqn. 23].

For comparison, $\beta$-functions vs. bare tune at zero intensity are also shown for the same stopband width of 0.020 (Fig. 1). An essential difference between the two cases is that in the space charge case, the stopband is curved. This is due to the nonlinear dependence of the space charge force on envelope size. In fact, both the horizontal and the vertical crossings of the 13/2 resonance show up in the space charge case, since the space charge force couples the two.

Calculations similar to those shown in Fig. 1 were also made for a smooth focusing case. No essential difference
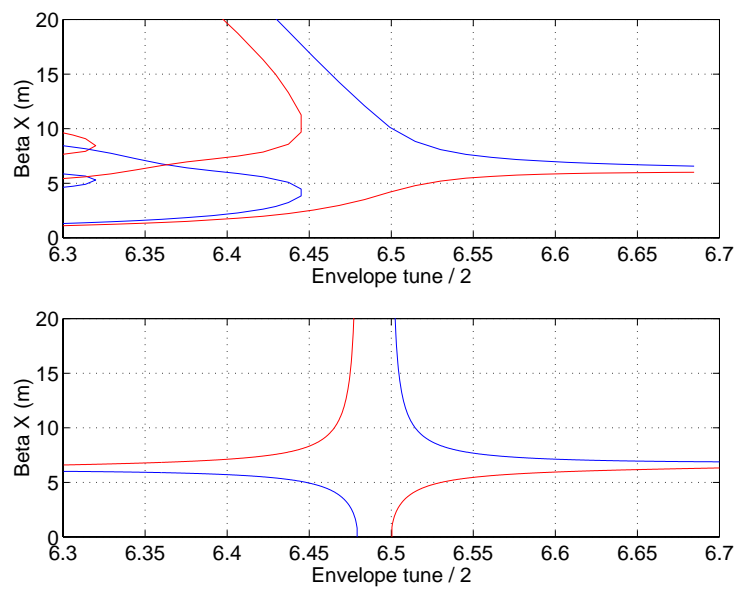

Figure 1: Matched $\beta$-function near the 13/2 resonance and with a stopband of 0.02 , vs. horizontal tune. The blue curve is for the location of the driving thin lens, and the red curve is for the location in the ring diametrically opposite to the thin lens. In the lower plot, envelope tune is changed by changing the bare tune at zero intensity. In the upper plot, the bare tune is left at 6.7, and the intensity increased: the left-most point where the envelope tune is 12.6 corresponds to an incoherent tune shift of 0.6 and a circulating current of $2.6 \mathrm{~A}$.

was evident. This demonstrates that the strong intrinsic resonances for tunes near 6 due to the 24-cell structure of the Booster lattice, did not play a role.

One can notice from Fig. 1 that at high intensity there are as many as 5 distinct matched beams. ACCSIM simulations were used to determine to what extent these solutions are realistic. One suspects that solutions far from the unperturbed $\beta$-functions depend on the beam distribution being $\mathrm{KV}$ to very high accuracy.

\section{SIMULATIONS}

An immediate problem encountered in ACCSIM simulations with the nominal tune $(6.7,6.8)$ is that for space charge tune shifts larger than the 0.1 split, emittance is continually exchanged between $x$ and $y$. This is the Montague resonance[5], and is probably a real effect. This moreover makes it impossible to fill the horizontal acceptance, which in the FNAL Booster is much larger than the vertical. This simulation result is in agreement with experience at the Booster. Nevertheless, for equal emittances, it is possible to avoid emittance-swapping by starting at zero intensity in ACCSIM and sweeping intensity at the rate of about .03 A per turn. Results are shown in Fig. 2

To test the validity of matches calculated by TRANSOPTR and shown in Fig. 1, accurately KV distributions were used in ACCSIM. To avoid the emittance-swapping phenomenon, the lattice was retuned to tunes $(5.7,6.8)$. For incoherent tune shifts less than about 0.29 , the matched beams grow only slightly in emittance, and stay matched over 100 turns. 


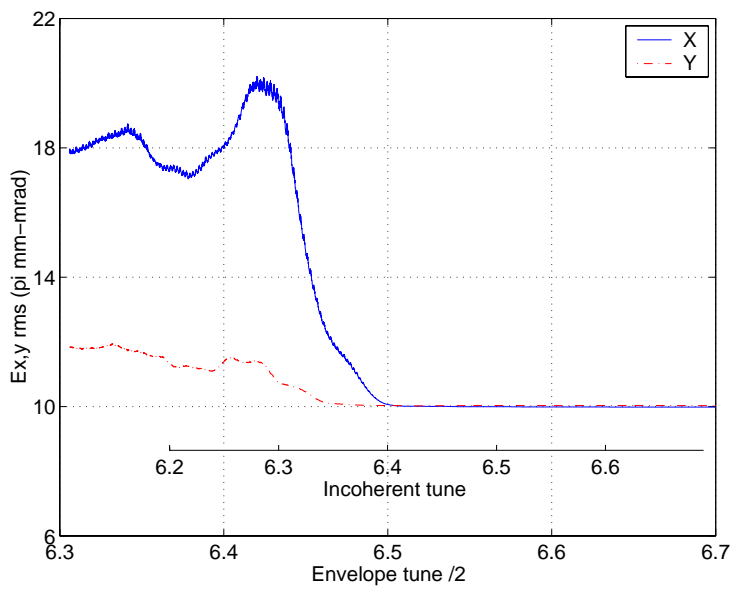

Figure 2: Emittance growth as the intensity is swept from 0 to $2.5 \mathrm{~A}$. This is plotted as a function of tune, so the curve proceeds from right to left. Resonance occurs at $I=1.3 \mathrm{~A}$, corresponding closely to when the envelope tune is 13 . The simulation used $10^{4}$ macro-particles.

As shown in Fig. 1, when the incoherent tune shift is larger than about 0.4 there is more than one matched solution of the envelope equation. We have tried several cases in this regime, where the matched $\beta$-functions are significantly different from the unperturbed case. In all cases, the distributions appear to stay matched initially, but then as numerical errors cause a deviation from $\mathrm{KV}$, emittance begins to grow dramatically. See Fig. 3 which corresponds to the case where the matched $\beta_{x}=26 \mathrm{~m}$, more then three times larger than the unperturbed $\beta_{x}=7.3 / \mathrm{m}$. (This case corresponds to the upper branch in Fig. 1.)

These results can be understood as follows. As the distributions deviate from KV, they are no longer matched. Rms beam sizes grow until the beam is large enough that the tune shift has fallen below threshold.

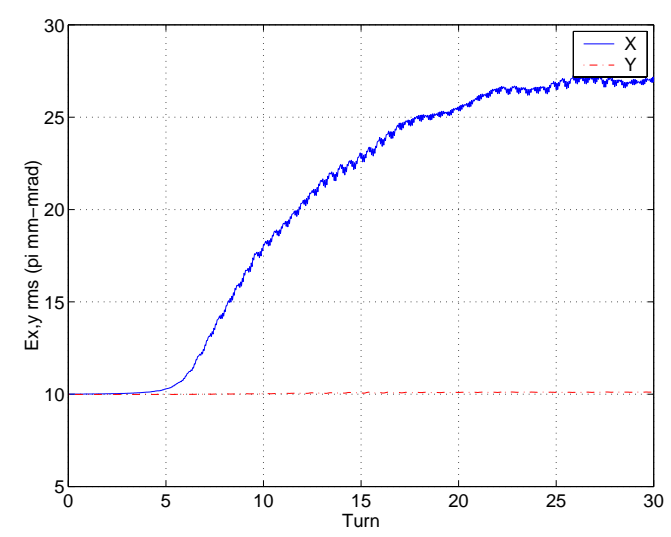

Figure 3: Emittance evolution of a matched KV distribution, at an intensity above that which is normally considered the threshold for the half-integer resonance. Bare tune is 5.7 , initial envelope tune $/ 2=5.4$.

\section{SUMMARY}

Macro-particle simulations have been performed. Starting with distributions which are matched as calculated by solving the envelope equations has been found to be an advantage in that emittance growth can be analyzed without the complicating feature of an initial mismatch.

An interesting result of these studies is that while matched solutions exist for intensities which are beyond the limit imposed by the envelope instability, these solutions cannot be accessed by increasing intensity slowly. Moreover, single turn injection of a beam that is matched but beyond the envelope instability only seems to be stationary for the artificial KV distribution.

This has practical implications for multiturn injection in storage rings. As well, for a bunched beam, where particles with large synchrotron amplitude circulate between regions of peak local current and low current, the space charge limit is likely imposed by the envelope instability. Even if the $1 / 2$-integer stopband is very small, for intensity beyond the limit, there will be some part of the bunch always on the envelope resonance, and this will cause emittance growth.

\section{ACKNOWLEDGMENTS}

We would like to thank H. Grote and R. Servranckx, respectively, for their kind permission to use input routines from MAD and optics/matrix routines from DIMAD to develop the enhanced version of ACCSIM used in this study.

Information re the FNAL booster was obtained in discussions with C. Ankenbrandt, M. Popovic, V. Shiltsev and C. Johnstone.

\section{REFERENCES}

[1] F.J. Sacherer: RMS Envelope Equations with Space Charge, IEEE Trans. Nucl. Sci. NS-18, p. 1105 (1971).

[2] M.S. de Jong and E.A.Heighway: A First Order Space Charge Option for TRANSOPTR, IEEE Trans. Nucl. Sci. NS30, p. 2666 (1983).

[3] R. Baartman: Low Energy Beam Transport Design Optimization for RIBs, to be published in Proc. ProRIB-2001, Calcutta, India.

[4] S.Y.Lee, H. Okamoto: Space-Charge Dominated Beams in Synchrotrons, Phys. Rev. Lett. 80 p. 5133 (1998).

[5] I. Hofmann, O. Boine-Frankenheim, J. Qiang, R. Ryne: Emittance Coupling in High Intensity Beams, these proceedings.

[6] R. Baartman, "Betatron Resonances with Space Charge," Workshop on Space Charge Physics in High Intensity Hadron Rings, Shelter Island NY, May 1998, AIP Conf. Proc. 448 p. 56.

[7] F.W. Jones, "Development of the ACCSIM Tracking and Simulation Code," Proc. 1997 Particle Accelerator Conference, Vancouver, 1997, p. 2597.

[8] J. Galambos et al., "ORBIT - A Ring Injection Code with Space Charge," Proc. 1999 Particle Accelerator Conference, New York, 1999, p. .3143 